\title{
Ultrasound Examination in Healthcare Facilities of Refugee Camps
}

J. Bydzovsky (Jan Bydzovsky), M. Jackulikova (Maria Jackulikova), H. Kalatova (Helena Kalatova), M. Dvorak (Marek Dvorak), K. Bulkova (Katerina Bulkova)

St. Elisabeth University of Healthcare and Social Work, Bratislava, SK

\section{E-mail address:}

bydzovsky@vszsp.cz

\section{Reprint address:}

Jan Bydzovsky

VSZaSP sv. Alzbety

Jiraskovy sady 240

26101 Pribram

$\mathrm{CZ}$

Source: Clinical Social Work and Health Intervention

Volume: 9

Issue: 1

Pages: $7-13$

Cited references: 3

\section{Reviewers:}

Daniel J. West

University of Scranton, Department of Health Administration and Human Resources, USA

Johnson Nzau Mavole

Catholic university of Eastern Africa, Nairobi, KE

\section{Key words:}

Ultrasound. Refugees. Abdominal Pain. Pregnancy. Field Healthcare Facilities.

\section{Publisher:}

International Society of Applied Preventive Medicine i-gap

CSWHI 2018; 9(1): 7 - 13; DOI 10.22359/cswhi_9_1_01 @ 2018 Clinical Social Work and Health Intervention

\section{Abstract:}

This article depicts the usage of ultrasound imaging in field healthcare facilities of St. Elisabeth University of Healthcare and Social Work in Bratislava - in two refugee camps in Greece during a one-week period when they were staffed by a Physician experienced in and licensed for ultrasound imaging. $16.2 \%$ of patients have been indicated by the Physician to be examined by ultrasound and $15.8 \%$ of these had 
abnormal findings. The majority of the examinations were indicated for pregnancy-related issues or abdominal pain. Despite this sample being too small we have clearly observed a great need for skills in obstetric ultrasound in this humanitarian project.

\section{Introduction}

Field healthcare facilities of St. Elisabeth University of Healthcare and Social Sciences in Bratislava ran in two refugee camps in Greece were one of many humanitarian projects of this university at present. This project ran since May 2016 and comprised two outpatient departments providing primary medical care similar to general practitioner for refugees in camps near towns Veroia and Alexandria in the Imathia Region of Northern Greece. These facilities were staffed by a Slovak team consisting of a Doctor (mainly with a specialty in general practice, infectious diseases or Pediatrics), a Pediatric Nurse and a Social Worker. This team employed translators from Arabic to English and was accompanied by Greek staff from KEELPNO (Hellenic Center for Disease Control \& Prevention), a Nurse and a driver, and cooperated with the Greek Army in whose former barracks the refugee camps were settled. Their inhabitants were refugees mainly from Syria on their journey to Europe. They were already registered by UNHCR and waiting in the camps for up to several months to be accepted by another European country to become their final destination.

In September 2017, the facilities looked after approximately 270 refugees in Veroia Camp and 300 refugees in Alexandria Camp. 24 of them (i.e. $4.2 \%$ of inhabitants) were pregnant females. The working hours of the clinics were at that time from 10 a.m. to 1 p.m. in Veroia Camp and from 2 p.m. to 5 p.m. in Alexandria Camp. The one hour in between was for the staff to move $25 \mathrm{~km}$ from one camp to the other. Each camp was visited every working day, from Monday to Friday, by roughly 10 to 15 patients.

Each facility was well-equipped for the medical care it provided. This included all necessary medicines both for enteral and parenteral administration and medical equipment such as blood pressure monitors, thermometers, a blood sugar monitor, a pulse oximeter, a CRP analyzer, a hemoglobin monitor, an otoscope, an ophthalmoscope, pregnancy tests, test kits for hepatitis and HIV, a nebulizer, a 12-lead ECG, an automated external defibrillator, a stretcher and a portable ultrasound. There was an ambulance car for the referrals of more serious cases to the hospitals and transfers of patients to and from their appointments at specialists.

The ultrasound machine was a Mindray, 15“ Portable Model DP-50 equipped with a $3.5 \mathrm{MHz}$ convex and a $7.5 \mathrm{MHz}$ linear multi-frequency probes capable of standard B-mode including tissue harmonic imaging (THI) and standard functions like measurements in B- and M-mode, estimation of gestational age in obstetric ultrasound etc. Unfortunately, the ultrasound machine was not much used for most of the time, because the majority of the Doctors were inexperienced in ultrasound imaging.

This article describes the usage of an ultrasound machine during a one-week period from $4^{\text {th }}$ to $8^{\text {th }}$ September 2017 when the facilities were staffed by an Emergency Physician skilled in ultrasound imaging and licensed for abdominal ultrasound imaging. 


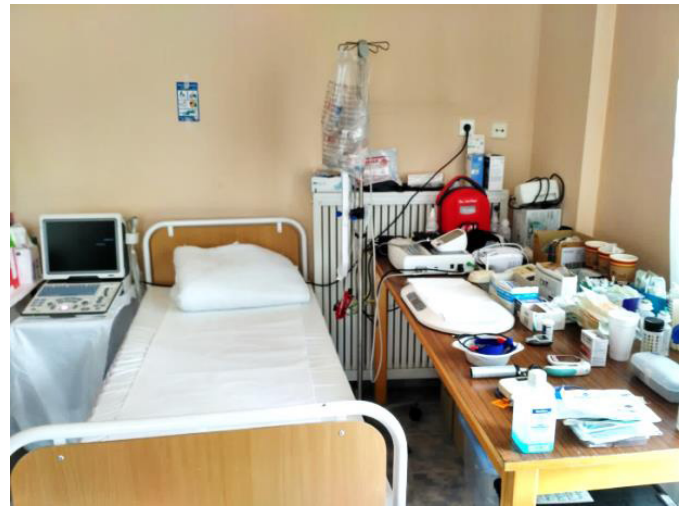

Fig. 1: View of the clinic in Veroia refugee camp.

\section{Material and Methods}

At that time, there were 123 visits in both the camps, most of them with minor problems and injuries, e.g. a sore throat, diarrhea, backache, rashes, insect bites etc. Patients coming with complaints such as abdominal pain were examined by ultrasound as they would be in our normal European "non-field" setting. Pregnant women were coming when it became known that there was a possibility of ultrasound check-up of their pregnancy. Most of these had never been examined by ultrasound in this pregnancy on their way to Europe.

There were 20 ultrasound examinations carried out for 19 patients (i.e. $16.2 \%$ one ultrasound exam in 6 visits) aged 3-60 years (mean $26 \pm 15.1$ years); 13 females aged 3-58 years (mean $26 \pm 11.7$ years); 6 males aged 14-60 (mean $34 \pm 17.6$ years) one of them had been examined twice. The summary of indications for the ultrasound examination is shown in the graph (Fig. 3). Although the sample is too small, it can provide some general impression: the majority was represented by abdominal pains and pregnancy-related problems including common check-ups - these obstetric screenings accounted for $40 \%$ of all ultrasound examinations.

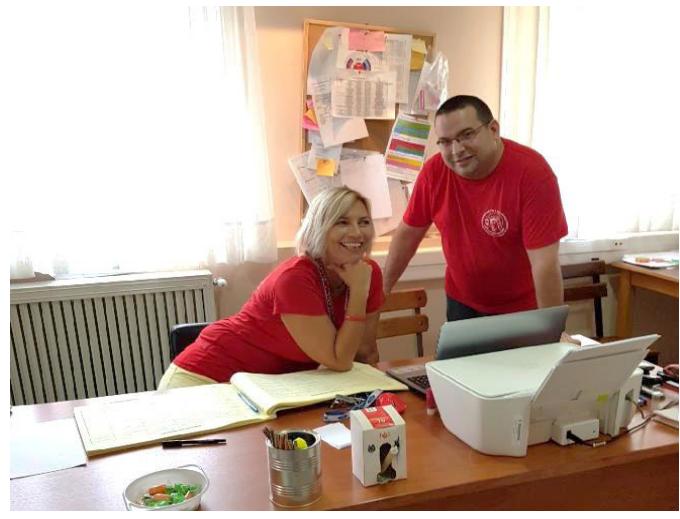

Fig. 2: Nurse Maria \& Dr.Jan Veroia Clinic.

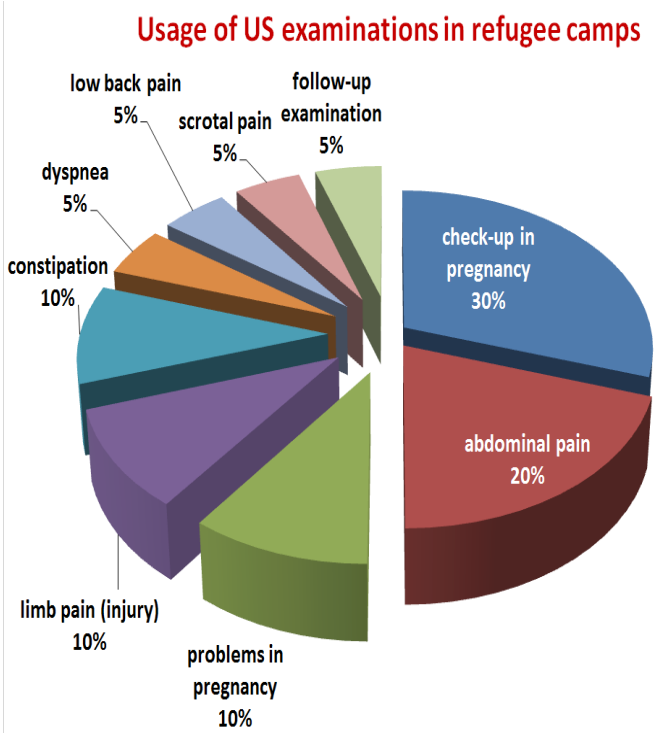

Fig. 3: Usage of ultrasound examinations in refugee camps.

The greatest need, in two fifths of the sample, was for obstetric ultrasound. Its goals were mainly to:

- confirm the pregnancy (gestational sac is visible from 3-5 weeks - first empty, then with a yolk sac and finally with an embryo from about 6 weeks),

- tell the number of embryos/fetuses,

- assess the viability of the embryo/fetus: detect cardiac action and measure the fetal heart rate using M-mode (FHR, 
around 100-120 bpm at 6 weeks, up to 9 weeks it increases to $120-180 \mathrm{bpm}$ ) and assess fetal movements,

- $\quad$ estimate the gestational age (GA) using a built-in program,

- describe the insertion of a placenta (visible from 10-12 weeks and may change as the lower uterine segment grows) to rule out placenta previa or to plan Caesarian section in advance. (The incidence lowers from about 5\% at 16-18 weeks to $0.5 \%$ at delivery.),

- assess the cervical length in $2^{\text {nd }}$ and $3^{\text {rd }}$ trimester and dilation (should be $>25 \mathrm{~mm}$ and not prematurely dilated if there is no insufficiency).

The determination of the sex of the fetus was not a goal of such examinations but serves to assure the mother that her baby is doing well and calm her mainly in case of abdominal pains etc. The parents should be told the sex of the fetus only when there is no doubt about it and they want to know it. Although it can be determined from about 12 weeks of gestational age, this also demands a good resolution of the ultrasound machine and an experienced operator. At any time if the findings are equivocal or the examination is technically difficult, a follow-up examination should be obtained.

The rest of the examinations were mainly common abdominal ultrasounds, but it is helpful if the physician is also experienced in basic echocardiography to rule out major disorders like pericardial effusion, RV enlargement and IVC dilation as signs of possible pulmonary embolism or signs of dehydration and hypovolemic shock or cardiac failure. So called "BLUE protocol" (ruling out pneumothorax and assessing the lung tissue) can be useful in dyspneic patients. Another possible usage is to examine long bones and muscles if a fracture or muscle tear are suspected after an injury to the limb. This is quite simple and can rule out the fracture by visualization of an intact cortical surface (in several planes in order not to miss any angulation) when there are clinical doubts. This procedure can lower the number of possibly problematic referrals to hospitals for X-ray imaging. It is useful mainly for examination of diaphysis of long bones of extremities, clavicle, ribs and skull. Another aim in limb pain is to rule out deep vein thrombosis. For this application, a color doppler (color flow mapping) mode is used as a standard, but the basic examination can be carried out in B-mode as well to confirm that all the great veins are anechoic and fully compressible. Anyway, for all these purposes and other examinations of superficial tissues, a linear probe is needed. The convex probe (often of lower frequency and therefore lower resolution) with a spacer made of an infusion bag or a glove filled with water is a considerably worse alternative if the linear probe is not available.

\section{Results}

Although most of the examinations were, of course, with normal results, 3 out of these 19 patients (i.e. $15.8 \%$ ) were with abnormal findings: a sludge in the gallbladder (without signs of inflammation) in abdominal pain, a liver cancer (suspected of hepatocellular carcinoma, Fig. 4) in abdominal pain and an abscess in scrotal pain (Fig. 5) that had been evacuated and another examination has been carried out during the follow-up (therefore there were 20 examinations together).

8 females have been examined because of their pregnancy. Two of them complained about abdominal pain, one about not feeling fetal movements and one was unsure if she was pregnant or not, the others came just to be checked-up. The GA has been estimated using a built-in program of the ultrasound using GS (gestational sac) or CRL 
(crown-rump length) in the first trimester and BPD (bi-parietal diameter) or FL (femur length) in later pregnancies and has been 12 weeks +0 days on average (mean 12 weeks +1 day) - see Fig. 6-9. The fetal heart rates being measured using M-mode are shown in Fig. 10-11.

Other interesting findings were during the previous mission of first two authors at Alexandria Camp when a young female came complaining about not feeling the movements of her baby in her late pregnancy (about 6 months). The ultrasound examination revealed there were no detectable movements of the fetus, no cardiac action, and moreover, signs of decomposition of the fetus, a collapse of the skull respectively, as shown in Fig. 10. She has been referred (in overall good condition, without signs of sepsis and/or vaginal discharge etc.) to the local hospital in Veroia and then transferred to another one in Thessaloniki. Another female complaining about lower back pain has been diagnosed nephrolithiasis when several kidney stones were revealed (Fig. 11). These were cases of the previous missions in these camps and are not included in the numbers presented above. We estimate that the utilization of ultrasound examinations and the distribution of cases during previous two one-week missions were similar.

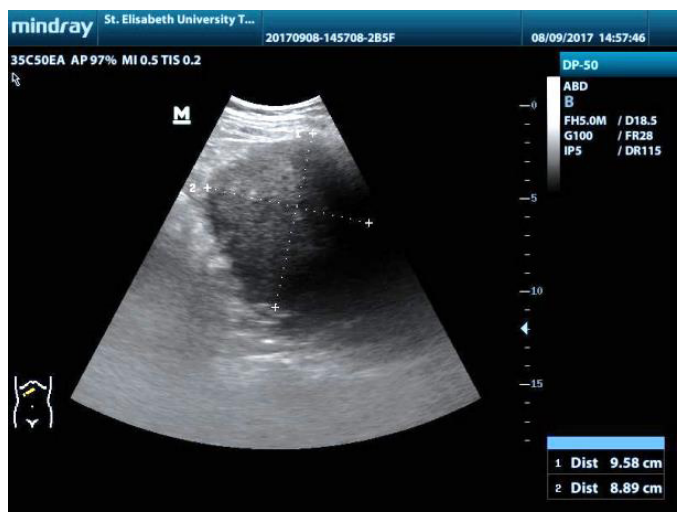

Fig. 4: liver cancer

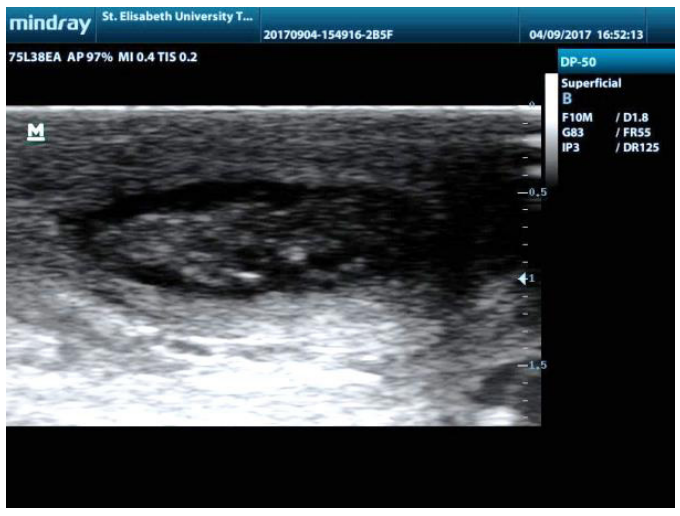

Fig. 5: scrotal abscess

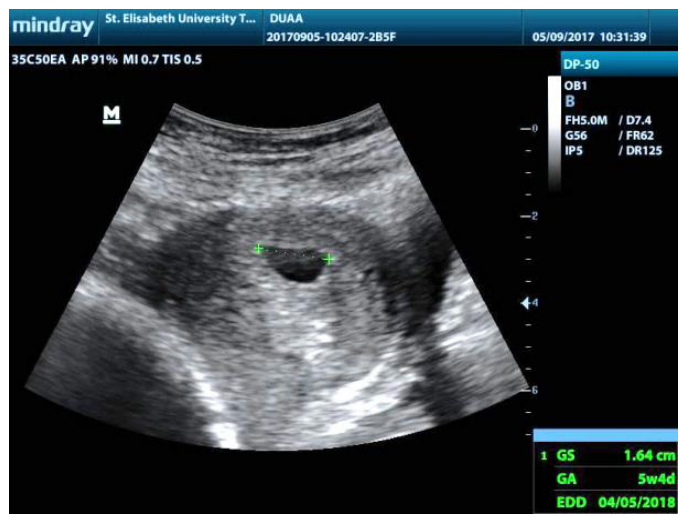

Fig. 6: estimation of GA using GS diameter

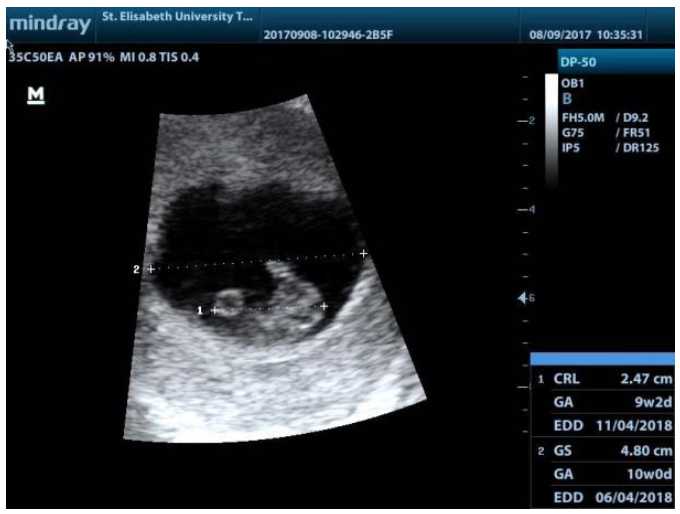

Fig. 7: estimation of GA using GS and CRL 


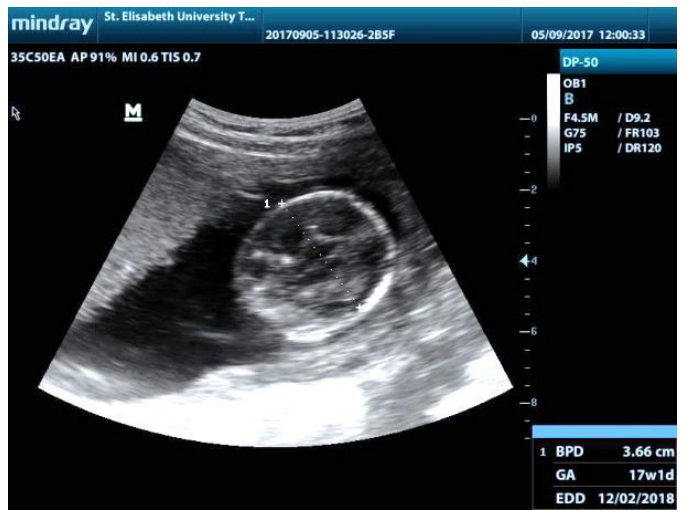

Fig. 8: estimation of GA using BPD

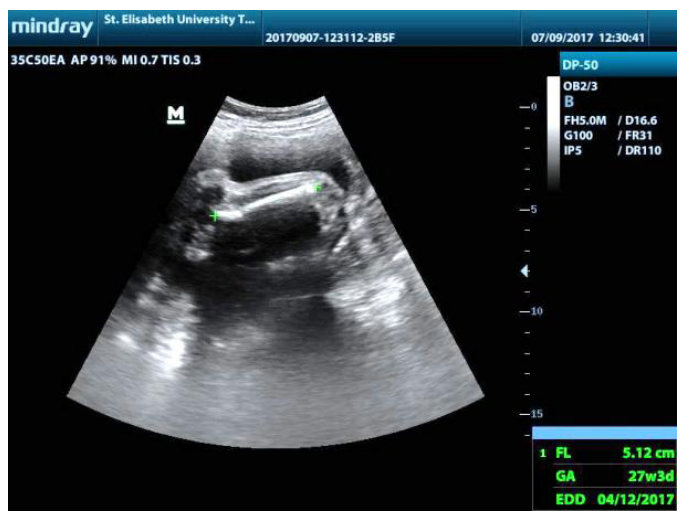

Fig. 9: estimation of GA using FL

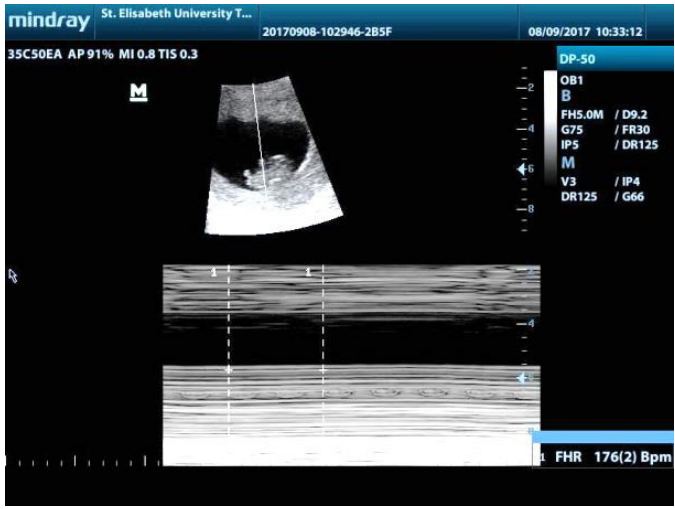

Fig. 10: estimation of FHR in embryo

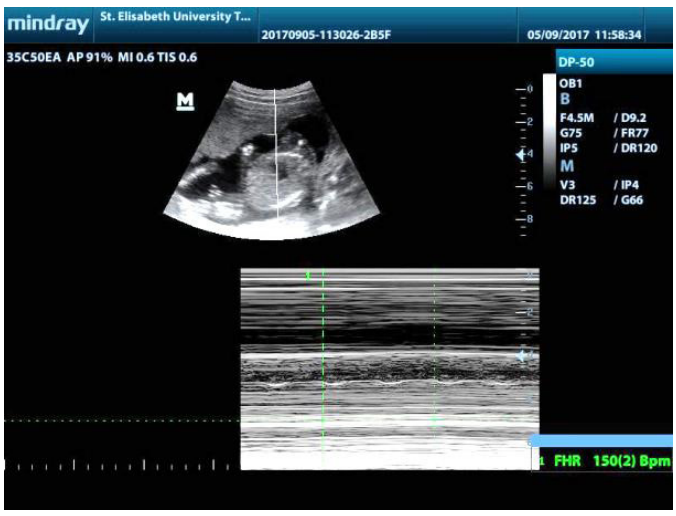

Fig. 11: estimation of FHR in fetus

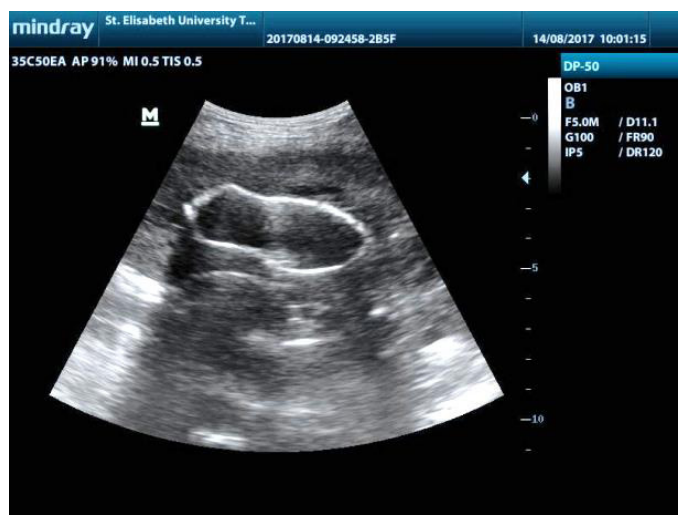

Fig. 12: collapsed skull in dead fetus

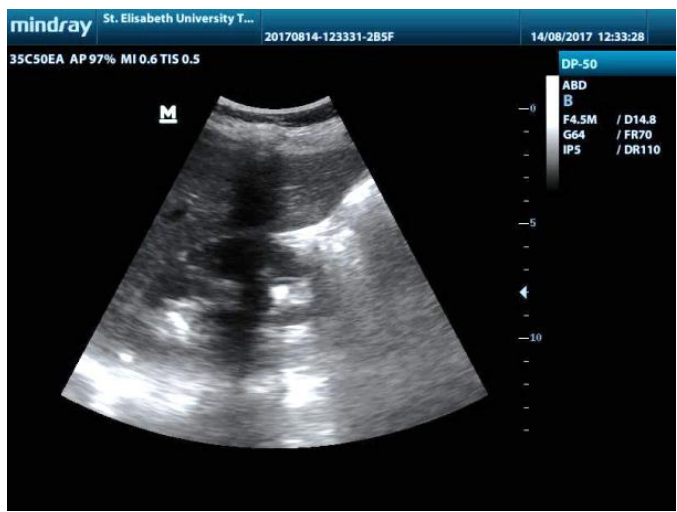

Fig. 13: nephrolithiasis in right kidney 
It must be said that we haven't noticed any bigger problems when examining Muslim women. They were shyer than non-Muslim female patients we are used to and tried to undress as little as possible but cooperative. Most of the examinations were performed in the presence of their husbands who stayed there automatically but did not interfere in any way.

\section{Conclusion}

The availability of ultrasound machine equipped with convex and linear probes and presence of a Physician capable of reliable ultrasound examination are advantageous aspects of field and other resource-limited healthcare facilities taking care of refugees who are mainly young people including pregnant females. We recommend obtaining skills and practice not only in abdominal ultrasound, vascular ultrasound and echocardiography but emphasize the need for at least basic knowledge in obstetric ultrasound.

\section{References}

1. MA O J, REARDON R F, SABBAJ A (2010) Emergency Ultrasonography. In: Tintinalli's Emergency Medicine: A Comprehensive Study Guide. $7^{\text {th }}$ Ed. McGraw-Hill, $\mathrm{p}$ e146-e163. ISBN 978-0071484800.

2. NOBLE V E, NELSON B, SUTINGCO AN (2007) Manual of Emergency and Critical Care Ultrasound. Cambridge: Cambridge University Press 249 p. ISBN 978-0-51135590-5.

3. SHAH S, PRICE D, BUKHMAN G, SHAH S, WROE E (2011) Manual of Ultrasound for Resource-limited Settings. Partners in Health 418 p. ISBN 978-0-98289698-0-8. 